\title{
CONTINUIDAD Y AJUSTES EN LA POLÍTICA EXTERIOR MEXICANA HACIA EL MUNDO ÁRABE Y EL MEDIO ORIENTE: LA AGENDA ECONÓMICA Y POLÍTICA
}

\author{
CONTINUITY AND ADJUSTMENTS IN MEXICAN \\ FOREIGN POLICY TOWARDS THE ARAB WORLD \\ AND THE MIDDLE EAST: \\ THE ECONOMIC AND POLITICAL AGENDA
}

\section{CONTINUITÉ ET MISE À JOUR DE LA POLITIQUE MEXICAINE VIS-Ä-VIS DU MONDE ARABE ET DU MOYEN-ORIENT: L'AGENDA ÉCONOMIQUE ET POLITIQUE}

\author{
Marta Tawil Kuri \\ El Colegio de México \\ mtawil@colmex.mx
}

RESUMEN: En el presente texto se explora el porqué y el cómo de las principales decisiones de México hacia Medio Oriente y el mundo árabe durante el sexenio del presidente Enrique Peña Nieto (2012-2018). Se sostienen dos argumentos: 1) los factores internos determinaron en buena medida el discurso y la práctica de la política exterior hacia esa región; 2) hubo continuidad en las líneas directrices y en los objetivos respecto al sexenio anterior; se registraron, sobre todo, ajustes en el empleo de los instrumentos de política exterior, así como en la implementación de las decisiones. El examen de estas continuidades y ajustes se desarrolla en torno a dos agendas interdependientes: la económica y la política.

Palabras clave: Medio Oriente; implementación; comercio; seguridad; continuidad; política exterior; México

ABstract: This text explores the why and the how of the main decisions taken by Mexico with regard to the Middle East and the Arab world during the presidency of President Enrique Peña Nieto (2012-2018). Two arguments are put forward: 1) internal factors largely determined foreign policy discourse and 
practice towards that region; 2) there was continuity in the principal guidelines and objectives with respect to the previous administration; above all, there were changes in the use of foreign policy instruments, as well as in the implementation of decisions. The examination of these continuities and changes is developed around two interdependent agendas: the economic and the political.

Keywords: Middle East; implementation; trade; security; continuity; foreign policy; Mexico

\section{Traducción de Gonzalo Celorio Morayta}

RÉsumÉ: Cet article cherche à expliquer pourquoi et comment le Mexique a pris ses décisions les plus importantes concernant le Moyen-Orient et le monde arabe sous le gouvernement d'Enrique Peña Nieto (2012-2018). Il propose deux idées: 1) les circonstances nationales ont dicté, dans une grande mesure, le discours et la pratique de la politique étrangère mexicaine envers ces territoires; 2) le fil conducteur et les buts de celle-ci n'ont pas changé par rapport au gouvernement précédent, malgré des variations dans le choix des instruments de cette politique et dans la manière de prendre les décisions. L'analyse de la continuité et des changements suit les deux agendas entrelacés de l'économie et de la politique.

Mots clefs: Moyen-Orient; mise en pratique; commerce; sécurité;

continuité; politique étrangère; Mexique.

Traducción de BERNARDo MABIRE

Fecha de recepción: diciembre de 2018

Fecha de aceptación: febrero de 2019 


\section{E}

$\mathrm{N}$ este artículo se exploran los principales acontecimientos que marcaron las relaciones de México con algunos países de Medio Oriente durante el gobierno de Enrique Peña Nieto (EPN). El objetivo es entender cómo ocurrieron y qué revelan de la política exterior mexicana. Se sostienen dos argumentos. En primer lugar, hubo factores internos que determinaron en buena medida el discurso y la práctica de la política exterior hacia esa región. Las motivaciones y acciones del gobierno de EPN deben, desde luego, situarse en el contexto del sistema político y económico internacional. Particularmente, las tensiones progresivas con Estados Unidos fueron un catalizador de la profundización de las relaciones comerciales de México con varios países de Medio Oriente. En segundo lugar, hay continuidad notable en las líneas directrices y los objetivos de la política hacia Medio Oriente en el gobierno de EPN respecto al sexenio anterior. Además de extender la presencia de México mediante la apertura de dos nuevas embajadas (en Jordania y en Qatar), ${ }^{1}$ se registraron ante todo importantes ajustes en el empleo de los instrumentos de la política exterior, principalmente los comerciales -que se ampliaron y profundizaron-, así como en la implementación de las decisiones. En este último plano, las preferencias y los estilos del presidente, sus cancilleres y otros funcionarios parecen haber tenido un peso relativo más visible que en el pasado, lo que a su vez se reflejó en los resultados. Debido a las grandes limitaciones de espacio, el presente examen se limita a dos ámbitos interdependientes: el económico y el de la agenda política y de seguridad.

${ }^{1}$ Antes de 2013, México contaba con representaciones diplomáticas en Arabia Saudita, Argelia, Egipto, Irán, Israel, Líbano, Marruecos, Kuwait, Emiratos Árabes Unidos (EAU)y en los territorios palestinos autónomos, dos de las cuales se abrieron durante el gobierno de Felipe Calderón (Kuwait, enero de 202, y EAU, diciembre de 2011). 


\section{AgENDA ECONÓMICA}

En este ámbito, destacan la asertividad y la coordinación intraburocrática; también se observa que las relaciones comerciales presentaron concentraciones sectoriales que pueden volverse estratégicas en el futuro.

\section{Turquía, Irán y Jordania}

De acuerdo con un diplomático mexicano entrevistado por la autora en diciembre de 2017, el acercamiento a Turquía se inició en el G-20, y la decisión de estimularlo fue del presidente Peña, informado por la Secretaría de Economía sobre el gran potencial comercial del país euroasiático. Con ese ímpetu, EPN realizó una visita oficial a ese país en diciembre de 2014. Para nuestro interlocutor: "El interés de institucionalizar la relación fue mutuo. Del lado mexicano, el sector agropecuario buscaba exportar a otros lugares que no fuera Estados Unidos [...] El Consejo de Relaciones Económicas Internacionales (DEIK, por sus siglas en turco) empezó a empujar en el tema automotriz". A iniciativa de la embajadora de México en Ankara, Martha Bárcena, se creó una comisión binacional en 2014 ( sin embargo, ésta se reunió por primera vez hasta 2017). Como parte de estas motivaciones, en febrero de 2015 el presidente Recep Tayyip Erdoğan reciprocó la visita de EPN. Tras sus encuentros en México, se efectuaron siete rondas de negociaciones para la firma de un Tratado de Libre Comercio (TLC). Sin embargo, hubo varias complicaciones. De acuerdo con el funcionario entrevistado, el particular estilo de negociación del equipo turco y la evolución de la política interna en Turquía, especialmente desde mediados de 2015, entorpecieron la relación. Los temas más complicados fueron el agropecuario y el textil. Las últimas tres rondas de negociación, a finales de 2015 y en 2016, revelaron el impasse al que habían llegado las discusiones sobre esos mercados. Se agregaron a esta situación la reforma constitu- 
cional destinada a reforzar el poder del presidente en el sistema político turco y, sobre todo, el intento de golpe de Estado el 15 de julio de 2016, todo lo cual terminó por llevarnos a quitar el pie del acelerador". ${ }^{2}$

Turquía se planteaba el objetivo de incrementar con México a 5000 millones de dólares el comercio bilateral. Esto es, Turquía percibe en México enorme potencial, como mercado y como puente hacia Estados Unidos, Centro y Sudamérica. Erhan Alma, secretario de la Cámara de Comercio e Industria México-Turquía, explicó que, ante la falta de energéticos como gas natural y petróleo en su país, un posible acuerdo comercial con México facilitaría a Turquía la compra de estos insumos mexicanos. ${ }^{3}$ En febrero de 2017, el ministro de Exteriores, Mevlüt Çavuşoğlu, inauguró oficialmente en la Ciudad de México la Agencia Turca de Colaboración y Coordinación (TIKA, por sus siglas en turco). Para Çavuşoğlu, gracias a esta oficina en México, se abrirán las puertas a América Latina. ${ }^{4}$

Con Irán -mercado de casi 80 millones de personas y una puerta de entrada a otros países de la región, como Pakistán, Afganistán e Iraq-, algunos elementos de la política de México pueden calificarse de novedosos en la medida en que, como otros gobiernos del globo, el gobierno mexicano buscó acercarse a este país por las expectativas generadas tras el

${ }^{2}$ En 2017, el Encargado de Negocios en México, Fikret Turkes, declaró que un grupo conocido como Casa Turca tenía vínculos con ese movimiento y que se había notificado de ello al gobierno mexicano. El funcionario entrevistado nos informó que la respuesta de México fue inequívoca: "México tiene legislación propia para perseguir delitos, su definición de terrorismo es distinta, y de cualquier manera el gobierno mexicano no tiene conocimiento de actividades ilícitas por parte de esas instituciones". José Carreño, "Turquía advierte sobre presencia de grupo radical islámico en México", El Heraldo de México, 13 de julio de 2017.

${ }^{3}$ Dainzú Patiño, "México abre puerta comercial a países del Medio Oriente", El Economista, 11 de junio de 2014.

${ }^{4}$ тікA actúa bajo los auspicios del Oficina del Primer Ministro. "Çavuşoğlu inaugura oficialmente la tika en Ciudad de México, donde mantiene conversaciones", TRT, 4 de febrero de 2017. 
levantamiento paulatino de sanciones económicas de la ONU, Estados Unidos y la Unión Europea. Entre otras cosas, dicho acuerdo iba a permitir transacciones bancarias, opciones de inversión extranjera -incluidos los sectores energético y automotriz-, de comercio (exportaciones e importaciones) y acceso a créditos. Desde septiembre de 2014, una delegación de funcionarios encabezada por el subsecretario de Relaciones Exteriores, embajador Carlos de Icaza, visitó la República Islámica con el objetivo de buscar oportunidad de negocios. Ambos gobiernos se comprometieron a impulsar las negociaciones en curso en materia de asistencia jurídica, colaboración en el combate a la delincuencia organizada transnacional, cooperación académico-diplomática, entre otros. En adelante, diversas misiones comerciales de empresarios iraníes y mexicanos realizaron visitas mutuas prolíficas. ${ }^{5} \mathrm{La}$ decisión de Washington, con el presidente Donald Trump, de cancelar el acuerdo nuclear con Irán e imponer nuevas sanciones en mayo de 2018, forzó a compañías extranjeras a cerrar sus negocios en el país y, en consecuencia, limitó en extremo el horizonte de los proyectos iraníes-mexicanos.

Jordania, país de la subregión del Levante, fue el otro objetivo de este gobierno. A decir de funcionarios de la secretaría y subsecretaría, a la decisión de abrir una embajada de México ante el reino Hachemí contribuyó un encuentro, en julio de 2013, entre el presidente Peña y el rey Abdallah en un foro multilateral; se dice que entre ambos hubo gran simpatía. En febrero de 2014, el rey visitó México por primera vez; en esa ocasión se suscribieron distintos instrumentos, entre ellos, los Términos de Referencia para la negociación de un TLC entre ambos países. Luego, el secretario José An-

5 "Misión comercial del comce en Irán", ProMéxico, 26 de septiembre de 2016; "Cancilleres de México e Irán se reúnen para reforzar relación bilateral", Terra Noticias, 14 de septiembre de 2016; Juan Antonio Cepeda, "Apuntes para una relación comercial entre México e Irán”, $N e$ xos, $1^{\circ}$ de enero de 2016; "México e Irán fortalecen vínculos y fomentan sus relaciones de comercio", Agencia Mexicana de Cooperación Internacional para el Desarrollo, 2 de diciembre de 2015. 
tonio Meade hizo una visita de trabajo a Jordania para dar seguimiento a los compromisos asumidos durante la visita del rey a México. ${ }^{6}$ Jordania depende casi en $100 \%$ del exterior para el abastecimiento de energía. Ante las crecientes dificultades de importación, el reino ha buscado diversificar sus fuentes de suministro.

\section{Israel y paises árabes del Golfo}

La agenda económica y el modelo de desarrollo de México se concretaron en una diplomacia económica particularmente asertiva hacia Israel y los países del Golfo. Un telón de fondo del interés de empujar el comercio con Israel -único país de la región con quien México tiene un TLC (2000)durante este sexenio fue el proyecto de la Alianza del Pacífico; éste pareció ser una de las principales motivaciones detrás de las políticas asertivas de Israel hacia México y América Latina en general. ${ }^{7}$ Aun así, el comercio bilateral continuó por debajo de su potencial; la tasa de crecimiento promedio anual de comercio total México-Israel es de $8.7 \%$, y fue de 942 millones de dólares en 2017. También se consolidó la colaboración tecnológica en materia de agua entre México e Israel, para hacer frente a los desafíos del campo mexicano. $^{8}$

6 "México negociará Tratado de Libre Comercio con Jordania", El Financiero, 6 de julio de 2014.

${ }^{7}$ El gobierno de Israel aprobó un plan de alrededor de 14.5 millones de dólares para fortalecer los lazos económicos con los miembros de la Alianza del Pacífico y otros países latinoamericanos. El plan incluye la cooperación con el Banco Interamericano de Desarrollo en un fondo para investigación industrial. Véase "Israel fortalecerá lazos económicos con América Latina", Unidos por Israel, 21 de mayo de 2014.

${ }^{8}$ Giovanni Bolaños, "Así ha convertido Israel el desierto en un imperio regional de agua potable", Administración de Comercio Exterior de Israel, 11 de diciembre de 2017. 
Por lo que a los países árabes respecta, desde el sexenio pasado el gobierno mexicano había buscado de manera más activa oportunidades de negocio para las empresas nacionales, sobre todo en la región del Golfo. ${ }^{9}$ En la continuidad de estos esfuerzos, Peña realizó una gira de trabajo a la península arábiga, del 17 al 21 de enero de 2016, que incluyó visitas de Estado a Arabia Saudita, Emiratos Árabes Unidos (EAU), Kuwait y Qatar. El artífice de esa gira fue José Antonio Meade quien, como secretario de Relaciones Exteriores, realizó una gira de trabajo en marzo de 2014 a Arabia Saudita, Qatar, Kuwait y Emiratos Árabes Unidos, y a Jordania en julio del mismo año. La visita se menciona en el cuarto informe de gobierno (2015-2016) con el calificativo de "histórica", al ser la primera de un presidente mexicano a los EAU y Qatar -desde el establecimiento de relaciones en 1975- y a Arabia Saudita y Kuwait desde el gobierno del presidente Luis Echeverría Álvarez (1970-1976). ${ }^{10}$ De acuerdo con el secretario de Economía, Ildefonso Guajardo, el gobierno mexicano aspiraba a que, como resultado de ésta, el comercio de México con los países árabes se triplicase en lo que restaba del sexenio. ${ }^{11}$

Los Emiratos Árabes Unidos fue el país con el que más se avanzó. La visita de Estado de Peña Nieto permitió iniciar el salto cualitativo en la relación con la firma del Acuerdo para la Promoción y la Protección Recíproca de las Inversiones (APPRI) y otros 12 instrumentos jurídicos y acuerdos interinstitucionales. Las prioridades de los EAU son la agricultura, las infraestructuras y logística (puertos), el turismo y las energías renovables. Dubái es, además, el punto de entrada para

${ }^{9}$ Hugo Salvatierra Arreguín, "Las empresas mexicanas que buscan conquistar el desierto árabe”, Forbes, 4 de julio de 2016.

${ }^{10}$ EPN viajó con los secretarios de Relaciones Exteriores, de Economía, de Energía, de Educación Pública, de Comunicaciones y Transportes, y los directores generales de Pemex, de Proméxico, de Bancomext y de Conacyt. Lo acompañó también un grupo de 19 empresarios.

${ }^{11}$ Rubén Migueles, "México triplicará comercio con países árabes en 3 años”, El Universal, 27 de enero de 2016. 
productos hacia la península arábiga, pero también para el norte de África y Asia Central. Su puerto se destaca por su tamaño y por su eficiencia logística. Tomando en cuenta esta realidad, se conversó sobre la conectividad aérea directa de vuelos comerciales entre los dos países. Asimismo, con el fin de fomentar más los vínculos bilaterales, en octubre de 2018 ambos gobiernos suscribieron un acuerdo recíproco de supresión de visas.

Aunque México no tiene aún preponderancia en ciertas importaciones del mundo árabe (por ejemplo, como Argelia o Siria que le compran a Brasil $90 \%$ del azúcar que consumen), lo que se hizo en este sexenio puede ser indicio de que hay sectores que simbolizan las interdependencias creadas por los flujos comerciales. Uno de ellos es el sector agroalimenticio. La caída histórica de los precios del petróleo reforzó la determinación de Emiratos de diversificar su economía y explorar nuevas oportunidades más allá de sus socios tradicionales (India, Estados Unidos, Unión Europea). Las exportaciones agroalimentarias totales de México a los EAU ascendieron a 20.3 millones de dólares durante los primeros cinco meses de 2018, lo que representó un crecimiento de $160 \%$ respecto al mismo periodo del año anterior, cuando las ventas nacionales registraron sólo 7.8 millones de dólares (según datos de la Consejería Agropecuaria facilitados por la Embajada de México en ese país del Golfo). ${ }^{12}$ El sector de la carne de res es uno de los más interesados en entrar a Medio Oriente, y comenzó a explorar las posibilidades antes del gobierno de Peña. En línea con los objetivos de diversificación de mercados, en 2014, ProMéxico encabezó una estrategia para la "promoción nacional e internacional de productos mexicanos con Certificación Halal 2015-2018".

México extendió su presencia en los países del Golfo al abrir una embajada en Qatar en marzo de 2014. Asimismo, en

12 "Las exportaciones agroalimentarias de México a Emiratos aumentan un 160\%”, El Correo del Golfo, 16 de noviembre de 2018. 
2014 la aerolínea Qatar Airways abrió una representación en el país. ${ }^{13}$ En el plano económico, es una excepción en la región: no tiene mucho petróleo, pero sí abundante gas natural licuado, que representa $90 \%$ de sus ingresos públicos. Asimismo, desde 1995, Doha ha desarrollado un activismo autónomo y propenso a ocupar la escena mediática mundial. Dado que el consumo de gas natural en México está creciendo más rápidamente que la producción, el acercamiento a Qatar puede responder a la necesidad de diversificar fuentes de ese recurso, sobre todo si se considera que en ese país hay grandes inversiones en tecnología para exportar gas natural a un precio más competitivo. Asimismo, con la gira de EPN se abrieron posibilidades a empresas mexicanas para proveer productos y servicios en la organización de la Copa Mundial de Futbol 2022. ${ }^{14}$

Los ejemplos expuestos confirman que se crearon sinergias entre ambos sectores empresariales. Además, el papel de las figuras políticas no se limitó al de los jefes de Estado; también en los distintos escalones burocráticos los lazos con Medio Oriente fueron fruto de voluntades individuales específicas. Por su parte, diversas instituciones y órganos fueron esenciales para la promoción comercial, como la Cámara Árabe Mexicana de la Industria y el Comercio (CAMIC), el Banco Mexicano de Comercio Exterior (Bancomext), ProMéxico, entre otros.

\section{Agenda POLÍ́tica y de SEgURIDAd}

El examen arroja un pragmatismo sobresaliente del gobierno mexicano, si bien aquél pareció apoyarse en gran medida

${ }^{13}$ Miriam Paredes, "Qatar Airways abrió una representación en México", Dinero en Imagen, 5 de noviembre de 2014.

14 Perla Pineda, "México y Qatar dinamizan su relación bilateral", El Economista, 22 de febrero de 2018; Engge Chavarría, "Mexicana, con pase para construir estadios en Catar", Heraldo de México, 14 de octubre de 2017. 
en una planificación a corto plazo y en visiones personalizadas, así como en la influencia de poderosos grupos de interés nacionales y transnacionales.

\section{Israel-territorios palestinos}

Entre 2013 y 2018, se profundizaron y ampliaron los vínculos con Israel. Hubo múltiples visitas oficiales mutuas, como del presidente Shimon Peres en el invierno de 2013 y del primer ministro Benjamin Netanyahu a México en septiembre de 2017, y la del presidente mexicano a Israel en el otoño de 2016. La manera en que la élite política con Enrique Peña se pronunció en el tema palestino-israelí se inscribe en la "política de equidistancia" del PRI, que también orientó las opciones de México bajo los gobiernos del PAN, con ajustes que se confirmaron en este sexenio. Con todo, la cosmovisión, el estilo y el peso político de los cancilleres Meade, RuizMassieu y Videgaray parecen elementos interesantes a indagar para estudios posteriores que expliquen los ajustes en la práctica, el discurso y la implementación de las decisiones de México en este tema. Más específicamente, podría inquirirse sobre la probabilidad de que la sucesión de tres cancilleres con diferentes pesos políticos haya podido reforzar la influencia del jefe de Estado y de su círculo más cercano (incluyendo poderes informales), lo que a su vez explique de manera decisiva algunas acciones y omisiones de política, o las improvisaciones.

En la cuestión palestina, el objetivo de empujar la imagen de México como "actor con responsabilidad global" era, a decir de todos los funcionarios de la secretaría y subsecretaría entrevistados por la autora, un interés y compromiso personal del secretario José Antonio Meade. Ese objetivo se acompañó del diálogo político bilateral con la Autoridad Palestina en Ramallah con dos visitas, una a finales de 2013 del embajador Carlos de Icaza, subsecretario de Relaciones Exteriores, y otra en mayo de 2017 del embajador Jorge Álvarez 
Fuentes, director general para África y Medio Oriente de la SRE. Con todo, el impacto de esos gestos pudo verse contrarrestado por el carácter discreto y equívoco del comunicado del gobierno mexicano acerca de la cruenta ofensiva israelí en Gaza durante el verano de 2014. ${ }^{15}$

La manera en que México lidió con dos complicados episodios de la agenda palestina-israelí a partir de 2016 deben inscribirse en un escenario interno e internacional sumamente difícil para México en el que, además, Meade dejó de estar al frente de cancillería para ser reemplazado, en agosto de 2015, por Claudia Ruiz-Massieu y luego por Luis Videgaray. El primer suceso ocurrió en octubre de 2016, cuando el Consejo Ejecutivo de la Conferencia General de la Organización de Naciones Unidas para la Educación, la Ciencia y la Cultura (UNESCO) aprobó una resolución para mantener la Ciudad Vieja de Jerusalén y sus muros en la lista del patrimonio de la humanidad en peligro, y criticaba a Israel por negar el acceso de los expertos a los lugares santos de la ciudad para verificar su conservación. ${ }^{16}$ Como en años anteriores, México votó a favor de la resolución. ${ }^{17}$ Esto es, el voto a favor fue producto de la política rutinaria de una declaración en la que la intención de México era, ante todo, afirmar posiciones generalizadas y colectivas en foros globales. ${ }^{18}$ Pero el voto se ganó

15 "México llama a Israel y Palestina a dejar hostilidades", Milenio, 14 de julio de 2014. Esto es particularmente notable al comparar el pronunciamiento de México con las declaraciones de condena de varios países en Latinoamérica, y con el hecho de que Argentina, Brasil, Chile, Ecuador y Perú llamaron a sus embajadores para consulta.

${ }^{16}$ La resolución, que lleva el título "Palestina ocupada", condena una larga lista de actos israelíes desfavorables a la población palestina. Fue aprobada por 24 votos a favor, 6 en contra y 26 abstenciones, que incluyeron a Francia, India y Argentina, países que en años anteriores habían votado a favor de resoluciones parecidas.

17 "Posición de México sobre la Decisión de la unesco relativa a Jerusalén”, Comunicado núm. 458, Secretaría de Relaciones Exteriores, 14 de octubre de 2016.

18 Tres años atrás, el 29 de junio de 2012, durante su 36 a sesión, el Comité de Patrimonio Mundial aprobó por 13 votos a favor (México in- 
inmediatamente el descontento de la comunidad judía en México, agraviada porque, desde su perspectiva, el documento negaba la relación del Monte del Templo en Jerusalén con el judaísmo. ${ }^{19} \mathrm{El}$ embajador de México ante la unesco, Andrés Roemer, quien es judío, declaró que el voto no había sido suyo, sino de México, y que él mismo estaba en contra. De hecho, abandonó el recinto de la UNESCo antes de que se conocieran los resultados finales de la votación. Además, difundió un documento de manejo interno de la SRE en el que se indicaba cómo votar. ${ }^{20}$ La polémica derivó en la destitución de Roemer. Al mismo tiempo, México buscó reconciliarse con la postura israelí al anunciar que se retractaba del voto anterior y solicitaba el cambio de su voto a uno de abstención. ${ }^{21}$ El cambio, explicó la SRE en un comunicado, "reitera el reconocimiento que el Gobierno mexicano otorga al vínculo innegable del pueblo judío con el patrimonio cultural ubicado en Jerusalén Oriental. Asimismo, refleja el enorme aprecio que México tiene por la comunidad judía”. ${ }^{22}$

cluido), 6 en contra y 2 abstenciones, la resolución con la que se inscribe el sitio "Lugar del nacimiento de Jesús: iglesia de la Natividad y la ruta de peregrinación, Belén” en la Lista de Patrimonio Mundial y en la Lista de Patrimonio Mundial en Peligro.

${ }^{19}$ El documento se refiere al lugar en Jerusalén solamente por su nombre árabe de Noble Santuario, una decisión semántica aprobada por la Junta Ejecutiva de unesco. Véase Silvia Garduño, "Critican voto sobre Jerusalén en UNESCo”, Reforma, 14 de octubre de 2016.

${ }^{20}$ Silvia Garduño, "Llama SRE a Andrés Roemer a consultas", Reforma, 14 de octubre de 2016; Silvia Garduño, "Instruyó subsecretaría voto sobre Jerusalén”, Reforma, 19 de octubre de 2016; Julio Hernández López, “Astillero", La Jornada, 19 de octubre de 2016.

${ }^{21}$ Patricia Muñoz Ríos, "La SRE cesa a representante ante la UNESCO", La Jornada, 18 de octubre de 2016; Iván E. Saldaña, "Le niegan a México el cambio de voto; Unesco rechaza cambio de postura”, Excélsior, 19 de octubre de 2016; "La unEsco aceptó el cambio de voto en el tema del Muro de los Lamentos: SRE” La Jornada, 20 de octubre de 2016.

22 "La SRE anuncia cambio de voto sobre preservación del patrimonio cultural de Jerusalén oriental”, Comunicado núm. 461, Secretaría de Re- 
Las vacilaciones ponen a la vista la ausencia de una política exterior de Estado, así como la falta de oficio vinculada a redes informales de poder. ${ }^{23}$ Apuntan, también, al tema más amplio de la implementación, el papel de sus actores y su grado de autonomía. En este caso, la implementación -conjunto de canales que sirven para traducir la política en práctica-24 muestra el peso de amiguismos, así como una brecha entre las intenciones de los políticos en la promulgación de una política específica y la forma en que la burocracia de la política exterior realmente la operacionaliza. Así por ejemplo, un documento de la sRe fechado el 12 de octubre demuestra que Andrés Roemer votó en el Consejo Ejecutivo de la UNESCo siguiendo las instrucciones de la Dirección General de Naciones Unidas de la Subsecretaria para Asuntos Multilaterales y Derechos Humanos de la cancillería mexicana. Sin embargo, algunos funcionarios entrevistados por la autora de este artículo lo negaron, destacando que Roemer no ofreció todos los elementos pertinentes a la Dirección General para Naciones Unidas para definir justamente la situación. ${ }^{25}$ Otras fuentes de la SRE indicaron que Roemer emitió

laciones Exteriores, 17 de octubre de 2016; Silvia Garduño, "Enreda a Cancillería voto en la Unesco", Reforma, 16 de octubre de 2016.

${ }^{23}$ El nombramiento de Roemer había sido objeto de impugnaciones por diversos legisladores, quienes cuestionaron que no tenía experiencia diplomática. En calidad de cónsul en San Francisco, se le acusó de seguir realizando actividades ajenas a su función, como la de organizar el encuentro la Ciudad de las Ideas, al lado del Grupo Salinas, en Puebla. Georgina Saldierna, "Relaciones Exteriores abre investigación por el caso Andrés Roemer en la UnEsco", La Jornada, 19 de octubre de 2016.

${ }^{24}$ Elisabetta Brighi y Christopher Hill, "Implementation and behavior", en Steve Smith et.al., Foreign Policy: Theories, Actors, and Cases, Oxford, University Press, 2012, pp. 147-167.

${ }^{25}$ De acuerdo con el diario Reforma, la cancillería precisó que la instrucción se giró en ese sentido porque Roemer no contestó a tres correos electrónicos que le envió la Subsecretaría para Asuntos Multilaterales y Derechos Humanos, previo a la votación. Una fuente mexicana familiarizada con el incidente dijo a la autora de este texto que Roemer sí informó a sus superiores mucho antes del voto. 
el voto sin consultar con la Subsecretaría para Asuntos Multilaterales, y sin advertir que países como Francia, España y Argentina cambiarían su posición tradicional y se abstendrían. Un diplomático dentro de la Secretaría confiaba a la autora en noviembre de 2016:

La resolución de la UNESCo era buena; ya teníamos un patrón de voto favorable que contó en esta ocasión también. La solicitud de cambio de voto hubiese sido innecesaria y la justificación del voto original relativamente fácil, de haber contado con la información suficiente. El tema de patrimonios culturales es muy importante para México, y varios funcionarios colegas y yo hemos constatado en persona lo que está ocurriendo en Jerusalén.

Un tema relacionado es el de la "complejidad rutinaria", que es la suma de numerosas microdecisiones adoptadas en el curso de la ejecución de una decisión, destinadas a traducir los imperativos políticos en acción y racionalizarlos como política. ${ }^{26}$ Así, por ejemplo, algunos funcionarios nos señalaron fallas de comunicación entre la canciller y la exjefa dela Oficina de la SRE, Ana Paola Barbosa (quien no quiso compartir su experiencia con la autora).

El segundo episodio se relaciona con el anuncio del presidente de Estados Unidos, Donald Trump, el 6 de diciembre de 2017, de que su país reconocería a Jerusalén como capital de Israel y ordenaría el traslado de la embajada de Tel Aviv a esa ciudad. Días después, el 21 de diciembre de 2017, la Asamblea General de la onu declaró por mayoría "nulo y sin efecto" el reconocimiento unilateral estadounidense. Nueve países votaron en contra, mientras que 35 se abstuvieron, entre ellos, México (en el continente americano también se abstuvieron Argentina, Canadá, Colombia y Panamá). Para explicar su abstención, México,

${ }^{26}$ Chris Alden y Ammon Aran, Foreign Policy Analysis: New approaches, Taylor and Francis, 2016, pp. 87- 106. 
a través de la SRE -ésta ya en manos de Luis Videgaray- reiteró su "firme convicción de que una solución política y pacífica del conflicto debe producirse mediante negociaciones directas, sin precondiciones [sic] entre las partes, que resuelvan los problemas sustantivos, incluido el estatus definitivo de Jerusalén [...], tal como fue pactado en los Acuerdos de Oslo y ratificado por diversas resoluciones". A su vez, el gobierno de Peña informó que México mantendría su embajada en Tel Aviv, de conformidad con las resoluciones del Consejo de Seguridad, así como las resoluciones pertinentes de la Asamblea General de la onu relativas al estatus de la Ciudad de Jerusalén.

En primer lugar, la mención "sin precondiciones" contradice la tradición de la diplomacia mexicana de defender las resoluciones y condiciones del derecho internacional y los organismos internacionales sobre la cuestión palestina-israelí. En segundo lugar, al ser un país que siempre ha defendido en el discurso "la solución de dos Estados" y la observancia del derecho internacional sobre el conflicto, México congeló la posibilidad de refrendar su compromiso internacional con el fortalecimiento del multilateralismo.

Por otro lado, desde una lectura estrictamente de tipo procesal y jurídica, podría decirse que México fue congruente al desistir del mensaje simbólico que cientos de países quisieron enviar a Washington (y Tel Aviv); en ese sentido, incluso podría argumentarse que su voto de abstención fue una muestra de autonomía al no votar ni a favor ni en contra. Por otra parte, la defensa de la existencia de dos Estados soberanos como solución al problema supone que Jerusalén sirva como capital de ambos, la parte oriental, de Palestina; la occidental, de Israel; sólo entonces será adecuado mover embajadas y emitir declaraciones de reconocimiento. La decisión de no posicionarse con claridad en esta polémica converge con el hecho de que México nunca ha dicho explícitamente que una parte de Jerusalén deba ser para la soberanía palestina, aunque siempre ha condenado la expansión de los asenta- 
mientos israelíes en Cisjordania y Jerusalén oriental como graves obstáculos a la paz. ${ }^{27}$

Es un hecho que siguen existiendo factores disuasivos de una actitud mexicana asertiva en el tema palestino-israelí: la influencia de los grupos de interés, la política exterior e interior de Estados Unidos, los desarrollos de los países latinoamericanos, la crisis social y política desde finales de 2014 en México, las preferencias del liderazgo mexicano, el papel del poder legislativo, entre otros.

En primer lugar, la insignia de Donald Trump como candidato a la presidencia fueron los insultos a México y a la inmigración, ante lo cual el gobierno de EPN optó por un acercamiento no contencioso; esto es, de apaciguamiento. A ello hay que agregar la alianza incondicional del candidato y luego presidente Trump y sus asesores, como Jared Kushner (muy cercano al canciller mexicano Videgaray), con Israel. Diplomáticos mexicanos en la región, así como funcionarios de la SRE, admiten que la dependencia económica hacia Estados Unidos sigue reduciendo el margen de maniobra de México; ello, junto con las presiones de congresistas estadounidenses en temas de la agenda bilateral, le impide ser autónomo en el tema palestino. El 6 de junio de 2016, cuatro meses antes del episodio de la UnEsco, la secretaria Claudia Ruiz-Massieu había pronunciado un discurso como invitada de honor a la 110 reunión anual del Comité Judío Americano (AJC, por sus siglas en inglés) que se celebró en Washington, DC. ${ }^{28}$ La secretaria insistió ante su audiencia en las interdependencias entre México y Estados Unidos, y en refutar ante la comunidad judío-estadounidense las acusaciones

27 Secretaría de Relaciones Exteriores, SRE, "México lamenta la decisión del Parlamento de Israel de aprobar una ley que legaliza asentamientos construidos en propiedad privada palestina”. Comunicado núm. 46, SRE, 7 de febrero de 2017.

28 "Discurso de la Canciller Claudia Ruiz Massieu, en el Comité JudíoAmericano (AJC) Global Forum”, Washington, DC, Embajada de México en Estados Unidos, 6 de junio de 2016. 
contra México del entonces candidato a la presidencia, Donald Trump. Rafael Fernández de Castro entrevé en este discurso una "nueva estrategia diplomática hacia Estados Unidos, que incluye cabildeo y campañas de imagen". ${ }^{29}$ Funcionarios de cancillería entrevistados por la autora subrayaron el fuerte cabildeo de los grupos sionista y textilero mexicano, así como el uso que el Congreso estadounidense hace del tema migratorio o de la seguridad como arma o recurso de negociación con México. Esto se relaciona, entonces, más con un segundo factor explicativo: la influencia de grupos de presión. Si bien no es fácil medirla con exactitud en la política exterior de México, parece seguro decir que el cabildeo sionista es mucho más fuerte, persistente y organizado que el árabe o palestino, si bien su efectividad nacional y transnacional suele depender de la acumulación de factores convergentes. ${ }^{30}$ En el caso de políticas que involucraron a Israel, el presidente y sus cancilleres, en especial Ruiz-Massieu y Videgaray, utilizaron la presencia comunitaria para justificar ciertas orientaciones de su política exterior.

La ausencia de contrapesos efectivos del poder legislativo es un tercer elemento a considerar. El peso de su voz en temas de Medio Oriente varía pero, en general, el congreso siguió la pauta de la presidencia en las políticas hacia la región, salvo en temas o momentos muy puntuales, como el que marcó la indignación por el apoyo de Netanyahu al muro de la frontera México-Estados Unidos.

Una cuarta variable, de naturaleza continental (americana), se relaciona con el apoyo entusiasta que algunos líderes centro y sudamericanos dieron a la decisión de Donald Trump de declarar a Jerusalén capital del Estado de Israel. ${ }^{31}$ En efec-

${ }^{29}$ Rafael Fernández de Castro, "La nueva estrategia diplomática hacia Estados Unidos", El Financiero, 10 de junio de 2016.

${ }^{30}$ Cecilia Baeza, "Le virage à droite en Amérique Latine et ses implications pour la Palestine", Agence Médias Palestine, 10 de enero de 2017.

31 Todos los países latinoamericanos, excepto, Colombia, Panamá y México, reconocieron el Estado de Palestina entre 2008 y 2013 (Colombia cambió su política y lo reconoció en agosto de 2018). El 31 de octubre 
to, la "década dorada" de las relaciones entre Palestina y América Latina se convirtió, desde aproximadamente 2015, en un símbolo de lo que la derecha denuncia como política exterior "ideológica". Esta evolución -relacionada con la toma de poder por parte de fuerzas derechistas en Sudamérica y su necesidad de ser aprobadas por Estados Unidos- pudo haber confirmado a la élite política en México la conveniencia de seguir defendiendo la neutralidad. ${ }^{32}$

En enero de 2017, el jefe del gobierno israelí, Benjamín Netanyahu, felicitó en su cuenta oficial de Twitter al presidente Trump por su idea de avanzar en la construcción del muro con México: "El presidente Trump tiene razón. Construí un muro en la frontera meridional de Israel. Detuvo toda la inmigración ilegal. Gran éxito. Gran idea”. Con una nota de protesta enviada al embajador de Israel, la SRE expresó su profunda "extrañeza, rechazo y decepción"; aclaró que México es amigo de Israel y "debe ser tratado como tal". ${ }^{33} \mathrm{El}$ embajador de Israel en México, Jonathan Peled, respondió que Netanyahu sólo compartía su experiencia en seguridad y no una postura sobre el muro. El primer ministro israelí aclaró que su país ha optado siempre por moderarse, "a pesar de que México vota regularmente contra Israel en las institucio-

de 2011, la unesco aprobó la adhesión de Palestina como Estado miembro de pleno derecho; México se abstuvo. El 29 de noviembre de 2012, la Asamblea General de las Naciones Unidas adoptó la Resolución 67/19 por medio de la cual se admitió al Estado de Palestina como Estado observador, estatus similar al que goza el Vaticano. En esa ocasión México votó a favor.

32 Cecilia Baeza, "Why did Latin America stop standing up for Palestine?", Al-Jazeera, 9 de junio de 2018. Paraguay fue el tercer país en trasladar su sede diplomática en Israel de Tel Aviv a Jerusalén, después de Estados Unidos y Guatemala, el 21 de mayo de 2018. El presidente sucesor revocó el 5 de septiembre la decisión. En abril de 2018, el Congreso Nacional de Honduras aprobó una moción para autorizar el traslado de su embajada, pero el poder ejecutivo aún no tomaba la decisión final.

33 Daniela Ivonne Méndez, "La comunidad judía responde al mensaje de Benjamín Netanyahu", The Huffington Post, 29 de enero de 2017. 
nes de la ONU". ${ }^{34}$ Posteriormente, la oficina del primer ministro y el Ministerio de Relaciones Exteriores de Israel emitieron una declaración para aclarar el tuit original, y el presidente israelí, Reuven Rivlin, finalmente se disculpó por el malentendido. Se dio vuelta a la página, y la visita a México de Benjamin Netanyahu meses después sirvió para enmendar el resabio y para que nuestro país buscara congraciarse más con Tel Aviv: así, dos meses después de la visita de Netanyahu, el secretario Luis Videgaray anunció en noviembre de 2017 que en adelante México "votaría en contra de las resoluciones de la onu que fueran contrarias a Israel". ${ }^{35}$ Esta actitud no era del todo inusual. ${ }^{36}$ Lo novedoso residió en el hecho de que un secretario en funciones lo planteara de forma abierta, pública, y ante representantes de otro gobierno en funciones.

\section{Cooperación en asuntos de defensa y seguridad}

En el área de la cooperación militar y de inteligencia, con Israel se registraron avances en la capacitación de policías y el uso efectivo de tecnologías. Uno de los casos más ilustrativos es el estado de Chiapas y los acuerdos que en la materia alcanzaron el secretario de Seguridad y Protección Ciudadana de ese estado, Jorge Luis Llaven Abarca, y el representante del Ministerio de Defensa de Israel en México, Honduras y Repú-

${ }^{34}$ Barak David, "Netanyahu Rejects Request by Minister to Apologize to Mexico Over Trump Wall Tweet”, Haaretz, 30 de enero de 2017.

35 "Mexico says it will henceforth vote with Israel in international bodies", The Times of Israel, 19 de noviembre de 2017. En la cuenta oficial de Twitter de Netanyahu se lee: "Thank you President of Mexico @EPN and Secretary of Foreign Affairs Videgaray for refusing to go along with one sided anti-Israel resolutions at the UN. Deeply value your friendship”, Twitter, @netanyahu, 14 de noviembre de 2017.

${ }^{36}$ Marta Tawil, "México ante Medio Oriente durante el gobierno de Felipe Calderón”, Foro Internacional, vol. LIII, 3-4, núms. 213-214 (julio-diciembre de 2013), pp. 667-706. 
blica Dominicana, Yaron Yugman. ${ }^{37}$ Por su parte, la Secretaría de Seguridad Pública del Distrito Federal adquirió un cargamento de armas de alto poder de fabricación israelí, con el fin de hacer frente a posibles incursiones del crimen organizado en la capital. ${ }^{38}$ Israel también está presente en la seguridad fronteriza mexicano-estadounidense. Desde hace algunos años, una empresa israelí de alta tecnología, Elkabetz, trabaja en el sur de Arizona a través de su filial Elbit Systems of America. ${ }^{39}$ A principios de septiembre de 2017, Elbit anunció un contrato para entregar aún más radares y torres de vigilancia para militarizar el área fronteriza entre México y Estados Unidos. La coalición de la sociedad civil palestina que lidera el movimiento global "Boicot, Desinversión y Sanciones (BDS) se ha movilizado contra estos contratos, así como contra las actividades de Cementos Mexicanos (Cemex). ${ }^{40}$ Asimismo, en el marco de la visita del primer ministro Netanyahu a México, en septiembre de 2017, el presidente Peña anunció que Israel asistiría los esfuerzos de Estados Unidos y México para impulsar proyectos de inversión y desarrollo en el llamado Triángulo Norte de América Central, en un esfuerzo por frenar la migración a Estados Unidos. ${ }^{41}$ México e Israel

37 "El ejército israelí entrenará a la policía de Chiapas", Excélsior, 8 de mayo de 2013.

${ }^{38}$ Hal Brands, "Los Zetas and Mexico's Transnational Drug War", World Politics Review, 25 de diciembre de 2009; Belén Fernández, "Death by «security»: Israel's services in Latin America", Al Jazeera, 7 de julio de 2018;. "Mexico's Chiapas Says It Receives Police Training From Israel, But Israel Embassy Denies It”, Fox News, 11 de enero de 2017.

${ }^{39}$ Todd Miller y Gabriel Matthew Schivone, "Why Is an Israeli Defense Contractor Building a «Virtual Wall»' in the Arizona Desert?", The Nation, 26 de enero de 2015.

40 "Trump Administration Hires Israeli Military Contractors to Build U.S.-Mexico Border Wall", Palestinian BDS National Committee, 28 de septiembre de 2017; "200+ Latin American Orgs Urge Mexican Multinational Cemex To End Complicity In Israeli Crimes", Palestinian BDS National Committee, 13 de julio de 2017.

${ }^{41}$ Alan Grabinsky, "Peña Nieto says Mexico accepted Israel's offer to help it and the U.S. develop Central America", Haaretz, 15 de septiembre de 2017; 
también anunciaron su intención de mejorar la cooperación en seguridad cibernética. ${ }^{42}$

Esta evolución, además de ser significativa de una evolución de las relaciones entre civiles y militares en estos países y en México, también muestra la importancia de los temas de defensa y seguridad para Tel Aviv y México en la dinámica de su relación con Washington.

\section{Conclusiones}

Explorar las relaciones de México con algunos países de Medio Oriente durante el gobierno de Enrique Peña revela mucho sobre la evolución de nuestra política exterior y el lugar de México en el mundo.

La agenda económica y el modelo de desarrollo de México se concretaron en una diplomacia económica particularmente asertiva. El papel de México de paladín del libre comercio impulsó mayores esfuerzos de institucionalización y coordinación. En los diversos escalones burocráticos, los lazos con Medio Oriente fueron fruto de voluntades individuales específicas dentro de la Dirección General para África y Medio Oriente de la cancillería, así como de los representantes diplomáticos mexicanos en esa región y de otros órganos de gobierno. Con todo, en un mundo tan incierto, fragmentado y peligroso como el actual, se necesitaba también invertir en peso político mundial, lo cual requería cuidar los recursos humanos y financieros, así como las atribuciones institucionales, de la Secretaría de Relaciones Exteriores. Por último, las contradicciones de la política de "equidistancia" en la cuestión palestina-israelí, o el activismo comercial, pudieron corresponder al objetivo de "desideologizar" la política exterior, para dar a México mayor legitimidad ante socios importantes; sin embargo, traicionaron la defensa de México del derecho

${ }^{42}$ Alan Grabinsky, "Netanyahu, Peña Nieto Meet, Agree to Bolster Israel-Mexico Cyber Security Ties”, Haaretz, 15 de septiembre de 2017. 
internacional, las resoluciones de la onu y el objetivo de ser "actor con responsabilidad global", además de que dejaron al país indefenso frente a diversas amenazas, como la violencia, los muros fronterizos y la militarización.

\section{Bibliografía}

Alden, Cris y Ammon Aran, Foreign Policy Analysis: New Approaches", Nueva York, Routledge, 2016.

Amorim Neto, Octavio y Andrés Malamud, "The policymaking capacity of foreign ministries in presidential regimes: a study of Argentina, Brazil and Mexico (1946-2015)", Latin American Research Review, 54, núm. 4, (2019) [en imprenta].

BaEza, Cecilia, "Why did Latin America stop standing up for Palestine?", Al-jazeera, 9 de junio de 2018.

Batza, Cecilia, "Le virage à droite en Amérique Latine et ses implications pour la Palestine", Agence Médias Palestine, 10 de enero de 2017.

Brands, Hal, "Los Zetas and Mexico's Transnational Drug War," World Politics Review, 25 de diciembre de 2009.

BrIgHI, Elisabetta y Christopher HiLl, "Implementation and behavior" en Steve Smith et al., Foreign Policy: Theories, Actors, and Cases, Oxford, University Press, 2012, pp. 147-167.

BRUn, Élodie, El cambio internacional mediante las relaciones Sur-Sur, México, El Colegio de México, 2018.

"Busca México ampliar exportaciones y oportunidades de negocio en el mercado de la península arábiga”, ProMéxico, Coordinación de comunicación institucional, 20 de febrero de 2018.

"Cancilleres de México e Irán se reúnen para reforzar relación bilateral", Terra Noticias, 14 de septiembre de 2016.

CARreño, José, "Turquía advierte sobre presencia de grupo radical islámico en México”, El Heraldo de México, 13 de julio de 2017.

CepedA, Juan Antonio, "Apuntes para una relación comercial entre México e Irán”, 1º de enero de 2016. 
Chavarría, Engge, "Mexicana, con pase para construir estadios en Catar”, Heraldo de México, 14 de octubre de 2017.

"Çavuşoğlu inaugura oficialmente la TıkA en Ciudad de México donde mantiene conversaciones", TRT, 4 de febrero de 2017.

DAvid, Barack, "Netanyahu Rejects Request by Minister to Apologize to Mexico Over Trump Wall Tweet", Haaretz, 30 de enero de 2017.

"Discurso de la Canciller Claudia Ruiz Massieu en el Comité JudíoAmericano (AJC) Global Forum", Washington, DC, Embajada de México en Estados Unidos, 6 de junio de 2016.

"El ejército israelí entrenará a la policía de Chiapas", Excélsior, 8 de mayo de 2013.

Fernández de Castro, Rafael, "La nueva estrategia diplomática hacia Estados Unidos”, El Financiero, 10 de junio de 2016.

FERNÁNDEZ, Belén, "Death by «security»: Israel's services in Latin America", Al Jazeera, 7 de julio de 2018.

Garduño, Silvia, "Critican voto sobre Jerusalén en unESCo", Reforma, 14 de octubre de 2016.

Garduño, Silvia, "Enreda a Cancillería voto en la unEsco", Reforma, 16 de octubre de 2016.

GARDUÑo, Silvia, "Instruyó subsecretaría voto sobre Jerusalén”, $R e^{-}$ forma, 19 de octubre de 2016.

Garduño, Silvia, "Llama sRe a Andrés Roemer a consultas", Reforma, 14 de octubre de 2016.

Grabinsky, Alan, "Peña Nieto says Mexico accepted Israel's offer to help it and the U.S. develop Central America", Haaretz, 15 de septiembre de 2017.

Grabinsky, Alan, "Netanyahu, Peña Nieto Meet, Agree to Bolster Israel-Mexico Cyber Security Ties”, Haaretz, 15 de septiembre de 2017.

Hernández López, Julio, “Astillero”, La Jornada, 19 de octubre de 2016.

"Israel fortalecerá lazos económicos con América Latina", Unidos por Israel, 21 de mayo de 2014.

Jiménez, Horacio, "Morena pedirá declarar persona non grata a Benjamín Netanyahu”, El Universal, 14 septiembre 2017. 
"La unesco aceptó el cambio de voto en el tema del Muro de los Lamentos: SRE" La Jornada, 20 de octubre de 2016.

"Las exportaciones agroalimentarias de México a Emiratos aumentan un 160\%”, El Correo del Golfo, 16 de noviembre de 2018.

Méndez, Daniela Ivonne, "La comunidad judía responde al mensaje de Benjamín Netanyahu”, The Huffington Post, 29 de enero de 2017.

“Mexico's Chiapas Says It Receives Police Training From Israel, But Israel Embassy Denies It”, Fox News, 11 de enero de 2017. "México e Irán fortalecen vínculos y fomentan sus relaciones de comercio", Agencia Mexicana de Cooperación Internacional para el Desarrollo, 2 de diciembre de 2015.

"México llama a Israel y Palestina a dejar hostilidades", Milenio, 14 de julio de 2014.

"México negociará Tratado de Libre Comercio con Jordania", El Financiero, 6 de julio de 2014.

"Mexico says it will henceforth vote with Israel in international bodies", The Times of Israel, 19 de noviembre de 2017.

"Mexico's Chiapas Says It Receives Police Training From Israel, But Israel Embassy Denies It”, Fox News, 11 de enero de 2017.

Migueles, Rubén, "México triplicará comercio con países árabes en 3 años", El Universal, 27 de enero de 2016.

Miller, Todd y Gabriel Matthew Schivone, "Why Is an Israeli Defense Contractor Building a «Virtual Wall» in the Arizona Desert?", The Nation, 26 de enero de 2015.

"Misión comercial del comce en Irán”, ProMéxico, 26 de septiembre de 2016.

MuÑoz Ríos, Patricia, "La SRE cesa a representante ante la UnESCo", La Jornada, 18 de octubre de 2016.

Ortiz, Guillermina, "Netanyahu niega haber mencionado a México por el muro de Trump", HuffPost, 30 de enero de 2017.

Paredes, Miriam, "Qatar Airways abrió una representación en México", Dinero e Imagen, 5 de noviembre de 2014.

Patiño, Dainzú, "México abre puerta comercial a países del Medio Oriente”, El Economista, 11 de junio de 2014.

Perthes, Volker, Arab Elites: Negotiating the Politics of Change, Boulder, Lynne Rienner Publishers, 2004. 
Pineda, Perla, "México y Qatar dinamizan su relación bilateral”, $E l$ Economista, 22 de febrero de 2018.

Ruiz Mondragón, Ariel, "Puentes posibles con el mundo árabe", Revista Comercio Exterior, 2018.

SALDAÑA, Iván E., "Le niegan a México el cambio de voto; Unesco rechaza cambio de postura", Excélsior, 19 de octubre de 2016.

Salvatierra Arreguín, Hugo, "Las empresas mexicanas que buscan conquistar el desierto árabe”, Forbes, 4 de julio de 2016.

Secretaría de Relaciones Exteriores, SRE, "Posición de México sobre la decisión de la unesco relativa a Jerusalén", Comunicado núm. 458, 14 de octubre de 2016.

Secretaría de Relaciones Exteriores, SRE, "La SRE anuncia cambio de voto sobre preservación del patrimonio cultural en Jerusalén Oriental”, Comunicado núm. 461, 17 de octubre de 2016. Secretaría de Relaciones Exteriores, sRe, "México lamenta la decisión del Parlamento de Israel de aprobar una ley que legaliza asentamientos construidos en propiedad privada palestina”. Comunicado núm. 46, sRe, 7 de febrero de 2017

Secretaría de Relaciones Exteriores, SRE, Secretaría de Gobernación, Segob, "México y Emiratos Árabes Unidos suprimen visas en pasaportes ordinarios", Comunicado conjunto SRE-SEGOB, 11 de octubre de 2018.

TAwIL, Marta, "México ante Medio Oriente durante el gobierno de Felipe Calderón”, Foro Internacional, vol. LIII, núms. 213-214 (2013), pp. 667-706.

"Trump Administration Hires Israeli Military Contractors to Build U.S.-Mexico Border Wall”, Palestinian BDS National Committee, 28 de septiembre de 2017.

VARGAS, Rosa Elvira, "Erdogan critica a Obama por asesinato de estudiantes", La Jornada, 13 de febrero de 2015.

Wallace, Arturo, "La huella en América Latina del Movimiento Gülen”, BBC Mundo, 25 de julio de 2016. 